\title{
Detecting Adverse Drug Reactions from Biomedical Texts With Neural Networks
}

\author{
Ilseyar Alimova \\ Kazan Federal University, \\ Kazan, Russia \\ ISAlimova@kpfu.ru
}

\author{
Elena Tutubalina \\ Kazan Federal University, \\ Kazan, Russia \\ Samsung-PDMI Joint AI Center, \\ PDMI RAS, St. Petersburg, Russia \\ elvtutubalina@kpfu.ru
}

\begin{abstract}
Detection of adverse drug reactions in postapproval periods is a crucial challenge for pharmacology. Social media and electronic clinical reports are becoming increasingly popular as a source for obtaining healthrelated information. In this work, we focus on extraction information of adverse drug reactions from various sources of biomedical textbased information, including biomedical literature and social media. We formulate the problem as a binary classification task and compare the performance of four state-of-the-art attention-based neural networks in terms of the F-measure. We show the effectiveness of these methods on four different benchmarks.
\end{abstract}

\section{Introduction}

Detection of adverse drug reactions (ADRs) in the post-marketing period is becoming increasingly popular, as evidenced by the growth of ADR monitoring systems (Singh et al., 2017; Shareef et al., 2017; Hou et al., 2016). Information about adverse drug reactions can be found in the texts of social media, health-related forums, and electronic health records. We formulated the problem as a binary classification task. The ADR classification task addresses two sub-tasks: (a) detecting the presence of ADRs in a textual message (messagelevel task) and (b) detecting the class of an entity within a message (entity-level task). In this paper, we focus on the latter task. Different from the message-level classification task, which aims to determine whether a textual fragment such as tweet or an abstract of a paper includes an ADR mention or not, the objective of the entity-level task is to detect whether a given entity (a single word or a multi-word expression) conveys adverse drug effect in the context of a message. For example, in "He was unable to sleep last night because of pain", the health condition 'pain' trigger insomnia. Meanwhile, in "after 3 days on this drug I was unable to sleep due to symptoms like a very bad attack of RLS", there is an entity 'unable to sleep' associated with drug use and can be classified as ADR.

Inspired by recent successful methods, we investigated various deep neural network models for entity-level ADR classification (Alimova and Tutubalina, 2018). Our previous experiments showed that Interactive Attention Neural network (IAN) (Ma et al., 2017) outperforms other models based on LSTM (Hochreiter and Schmidhuber, 1997). In this paper, we continue our study and compare IAN with the following attention-based neural networks for entity-level ADR classification: (i) Attention-over-Attention (AOA) model (Huang et al., 2018); (ii) Attentional Encoder Network (AEN) (Song et al., 2019); (iii) Attention-based LSTM with Aspect Embedding (ATAE-LSTM) (Wang et al., 2016). We conduct extensive experiments on four benchmarks which consist of scientific abstracts and user-generated texts about drug therapy.

\section{Related Work}

Different approaches are utilized to identify adverse drug reactions (Sarker et al., 2015; Gupta et al., 2018b; Harpaz et al., 2010). First works were limited in the number of study drugs and targeted ADRs due to limitations of traditional lexicon-based approaches (Benton et al., 2011; Liu and Chen, 2013). In order to eliminate these shortcomings, rule-based methods have been proposed (Nikfarjam and Gonzalez, 2011; Na et al., 2012). These methods capture the underlying syntactic and semantic patterns from social media posts. Third group of works utilized popular machine learning models, such as support vec- 
tor machine (SVM) (Liu and Chen, 2013; Sarker et al., 2015; Niu et al., 2005; Bian et al., 2012; Alimova and Tutubalina, 2017), conditional random fields (CRF) (Aramaki et al., 2010; Miftahutdinov et al., 2017), and random forest (RF) (RastegarMojarad et al., 2016). The most popular handcrafted features are n-grams, parts of speech tags, semantic types from the Unified Medical Language System (UMLS), the number of negated contexts, the belonging lexicon based features for ADRs, drug names, and word embeddings (Dai et al., 2016). One of the tracks of the shared task SMM4H 2016 was devoted to ADR classification on a tweet level. The two best-performing systems applied machine learning classifier ensembles and obtained $41.95 \%$ F-measure for ADR class (Rastegar-Mojarad et al., 2016; Zhang et al., 2016). Two other participants utilized SVM classifiers with different sets of feature and obtained $35.8 \%$ and 33\% F-measure (Ofoghi et al., 2016; Jonnagaddala et al., 2016). During SMM4H 2017, the best performance was achieved by SVM classifiers with a variety of surface-form, sentiment, and domain-specific features (Kiritchenko et al., 2018). This classifier obtained $43.5 \%$ F-measure for 'ADR' class. Sarker and Gonsales outperformed these result utilizing SVM with a more rich set of features and the tuning of the model parameters and obtained 53.8\% F-measure for ADR class (Sarker and Gonzalez, 2015). However, these results are still behind the current state-ofthe-art for general text classification (Lai et al., 2015).

Modern approaches for the extracting of ADRs are based on neural networks. Saldana adopted $\mathrm{CNN}$ for the detection of ADR relevant sentences (Miranda, 2018). Huynh T. et al. applied convolutional recurrent neural network (CRNN), obtained by concatenating $\mathrm{CNN}$ with a recurrent neural network (RNN) and CNN with the additional weights (Huynh et al., 2016). Gupta S. et al. utilized a semi-supervised method based on co-training (Gupta et al., 2018a). Chowdhury et al. proposed a multi-task neural network framework that in addition to ADR classification learns extract ADR mentions (Chowdhury et al., 2018).

Methods for sentiment analysis are actively adopted in the medical domain as well as in other domains (Serrano-Guerrero et al., 2015; Rusnachenko and Loukachevitch, 2018; Ivanov et al., 2015; Solovyev and Ivanov, 2014). In the field of aspect-level sentiment analysis, neural networks are popularly utilized (Zhang et al., 2018). Ma et al. proposed Interactive Attention Network which interactively learns attentions in the contexts and targets, and generates the representations for targets and contexts separately (Ma et al., 2017). The model compared with different modifications of Long Short Term Memory (LSTM) models and performed greatest results with $78.6 \%$ and $72.1 \%$ of accuracy on restaurant and laptop corpora respectively. Song et al. introduced Attentional Encoder Network(AEN) (Song et al., 2019). AEN eschews recurrence and employs attention based encoders for the modeling between context and target. The model obtained $72.1 \%$ and $69 \% \mathrm{f}$ accuracy on restaurant and laptop corpora respectively. Wang et al. utilized Attention-based LSTM, which takes into account aspect information during attention (Wang et al., 2016). This neural network achieved $77.2 \%$ and $68.7 \%$ of accuracy restaurant and laptop corpora respectively. The Attentionover-Attention neural network proposed by Huang et al. models aspects and sentences in a joint way and explicitly captures the interaction between aspects and context sentences (Huang et al., 2018). This approach achieved the best results among the described articles wit $81.2 \%$ and $74.5 \%$ of accuracy on restaurant and laptop corpora.

To sum up this section, we note that there has been little work on utilizing neural networks for entity-level ADR classification task. Most of the works used classical machine learning models, which are limited to linear models and manual feature engineering (Liu and Chen, 2013; Sarker et al., 2015; Niu et al., 2005; Bian et al., 2012; Alimova and Tutubalina, 2017; Aramaki et al., 2010; Miftahutdinov et al., 2017; RastegarMojarad et al., 2016). Most methods for extracting ADR so far dealt with extracting information from the mention itself and a small window of words on the left and on the right as a context, ignoring the broader context of the text document where it occurred (Korkontzelos et al., 2016; Dai et al., 2016; Alimova and Tutubalina, 2017; Bian et al., 2012; Aramaki et al., 2010). Finally, in most of the works experiments were conducted on a single corpus.

\section{Corpora}

We conducted our experiments on four corpora: CADEC, PsyTAR, Twitter, TwiMed. Further, we 
briefly describe each dataset.

CADEC CSIRO Adverse Drug Event Corpus (CADEC) consists of annotated user reviews written about Diclofenac or Lipitor on askapatient.com (Karimi et al., 2015). There are five types of annotations: 'Drug', 'Adverse effect', 'Disease', 'Symptom', and 'Finding'. We grouped diseases, symptoms, and findings as a single class called 'non-ADR'.

PsyTAR Psychiatric Treatment Adverse Reactions (PsyTAR) corpus (Zolnoori et al., 2019) is the first open-source corpus of user-generated posts about psychiatric drugs taken from AskaPatient.com. This dataset includes reviews about four psychiatric medications: Zoloft, Lexapro, Effexor, and Cymbalta. Each review annotated with 4 types of entities: adverse drug reactions, withdrawal symptoms, drug indications, sign/symptoms/illness.

TwiMed TwiMed corpus consists of sentences extracted from PubMed and tweets. This corpus contains annotations of diseases, symptoms, and drugs, and their relations. If the relationship between disease and drug was labeled as 'Outcomenegative', we marked disease as ADR, otherwise, we annotate it as 'non-ADR' (Alvaro et al., 2017).

Twitter Twitter corpus include tweets about drugs. There are three annotations: 'ADR', 'Indication' and 'Other'. We consider 'Indication' and 'Other' as 'non-ADR' (Nikfarjam et al., 2015).

Summary statistics of corpora are presented in Table 1. As shown in this table, the CADEC and PsyTAR corpora contain a much larger number of annotations than the TwiMed and Twitter corpora.

\section{Models}

\subsection{Interactive Attention Network}

The Interactive Attention Network (IAN) network consists of two parts, each of which creates a representation of the context and the entity using the vector representation of the words and the LSTM layer (Ma et al., 2017). The obtained vectors are averaged and used to calculate the attention vector. IAN uses attention mechanisms to detect the important words of the target entity and its full context. In the first layer of attention, the vector of context and the averaged vector of the entity and in the second, the vector of the entity and the averaged vector of context are applied. The resulting vectors are concatenated and transferred to the layer with the softmax activation function for classification.

\subsection{Attention-over-Attention}

Attention-over-Attention (AOA) model was introduced by Huang et al. (Huang et al., 2018). This model consists of two parts which handle left and right contexts, respectively. Using word embeddings as input, BiLSTM layers are employed to obtain hidden states of words for a target and its context, respectively. Given the hidden semantic representations of the context and target the attention weights for the text is calculated with AOA module. At the first step, the AOA module calculates a pair-wise interaction matrix. On the second step, with a column-wise softmax and row-wise softmax, the module obtains targetto-sentence attention and sentence-to-target attention. The final sentence-level attention is calculated by a weighted sum of each individual targetto-sentence attention using column-wise averaging of sentence-to-target attention. The final sentence representation is a weighted sum of sentence hidden semantic states using the sentence attention from AOA module.

\subsection{Attentional Encoder Network}

The Attentional Encoder Network (AEN) eschews complex recurrent neural networks and employs attention based encoders for the modeling between context and target (Song et al., 2019). The model architecture consists of four main parts: embedding layer, attentional encoder layer, targetspecific attention layer, and output layer. The embedding layer encodes context and target with pre-trained word embedding models. The attentional encoder layer applies the Multi-Head Attention and the Point-wise Convolution Transformation to the context and target embedding representation. The target-specific attention layer employs another Multi-Head Attention to the introspective context representation and context-perceptive target representation obtained on the previous step. The output layer concatenates the average pooling outputs of previous layers and uses a fully connected layer to project the concatenated vector into the space of the targeted classes.

\subsection{Attention-based LSTM with Aspect Embedding}

The main idea of Attention-based LSTM with Aspect Embedding (ATAE-LSTM) is based on ap- 
Table 1: Summary statistics of corpora.

\begin{tabular}{|l|c|c|c|c|}
\hline Corpus & Documents & ADR & non-ADR & Max sentence length \\
\hline CADEC (Karimi et al., 2015) & 1231 & 5770 & 550 & 236 \\
PsyTAR (Zolnoori et al., 2019) & 891 & 4525 & 2987 & 264 \\
TwiMed-Pubmed (Alvaro et al., 2017) & 1000 & 264 & 983 & 150 \\
TwiMed-Twitter (Alvaro et al., 2017) & 637 & 329 & 308 & 42 \\
Twitter (Nikfarjam et al., 2015) & 645 & 569 & 76 & 37 \\
\hline
\end{tabular}

pending the input aspect embedding into each context word input vector (Wang et al., 2016). The concatenated vectors are fed to the LSTM layer in order to obtain the hidden semantic representations. With the resulting hidden states and the aspect embedding, the attention mechanism produces an attention weight vector and a weighted hidden representation, which is applied for final classification.

\section{Experiments}

In this section, we compare the performance of the discussed neural networks with Interactive Attention Neural Network.

\subsection{Settings}

We utilized vector representation trained on social media posts from (Miftahutdinov et al., 2017). Word embedding vectors were obtained using word2vec trained on a Health corpus consists of 2.5 million reviews written in English. We used an embedding size of 200, local context length of 10 , the negative sampling of 5 , vocabulary cutoff of 10, Continuous Bag of Words model. Coverage statistics of word embedding model vocabulary: CADEC - 93.5\%, Twitter - 80.4\%, PsyTAR $54 \%$, TwiMed-Twitter $-81.2 \%$, TwiMed-Pubmed $-76.4 \%$. For the out of vocabulary words, the representations were uniformly sampled from the range of embedding weights. We used a maximum of 15 epochs to train IAN and ATAE-LSTM and 30 epochs to train AEN and AOA on each dataset. We set the batch size to 32 for each corpus. The number of hidden units for LSTM layer is 300 , the learning rate is $0.01,12$ regularization is 0.001 . We applied the implementation of the model from this repository ${ }^{1}$.

\subsection{Experiments and Results}

All models were evaluated by 5-fold crossvalidation. We utilized the F-measure to evaluate

\footnotetext{
${ }^{1}$ https://github.com/songyouwei/ABSA-PyTorch
}

the quality of the classification.

The results are presented in Table 2. The results show that IAN outperformed other models on all corpora. IAN obtained the most significant increase in results compared to other models on Cadec and Twitter-Pubmed corpora with $81.5 \%$ and $87.4 \%$ of the macro F-measures, respectively. We assume that the superiority of the IAN results in comparison with other models is due to the small number of parameters being trained and the small size of the corpora.

The AOA model achieved the second-place result on all corpora except Twitter. The AOA results for PsyTAR $(81.5 \%)$ and Twimed-Twitter (79.5\%) corpora state on par with IAN model, while for the rest corpora, the results are significantly lower. This leads to the conclusion that the model is unstable for highly imbalanced corpora.

The ATAE-LSTM model with $78.6 \%$ of macro F-measure outperformed AEN and AOA models results on Twitter corpora and achieved comparable with AOA results on Twimed-Pubmed corpora $(80.1 \%)$. This result shows that ATAE-LSTM applicable to a small size imbalanced corpora.

The AEN model achieved comparable with other models results on PsyTAR (80.2\%) corpora and significantly lower results on Twitter $(66.7 \%)$, Cadec (49\%) and Twimed-Pubmed (74.3\%) corpora. $72.4 \%$ of F-measure on Twimed-Twitter corpus states on par with the ATAE-LSTM model $(73.5 \%)$. This leads to the conclusion that the presence of multiple attention layers did not give the improvement in results.

\section{Conclusion and Feature Research Directions}

We have performed a fine-grained evaluation of state-of-the-art attention-based neural network models for entity-level ADR classification task. We have conducted extensive experiments on four benchmarks. Analyzing the results, we have found that that increasing the number of attention layers 


\begin{tabular}{|l|c|c|c|c|c|}
\hline Model & Twitter & Cadec & PsyTAR & Twimed-Twitter & Twimed-PubMed \\
\hline IAN & .794 & $\mathbf{. 8 1 5}$ & $\mathbf{. 8 1 7}$ & $\mathbf{. 8 1 9}$ & .874 \\
AEN & .667 & .490 & .802 & .742 & .743 \\
AOA & .752 & .752 & .815 & .795 & .803 \\
ATAE-LSTM & .786 & .702 & .807 & .735 & .801 \\
\hline
\end{tabular}

Table 2: Macro F-measure classification results of the compared methods for each datasets.

did not give an improvement in results. Addition an aspect vector to the input layer also did not give significant benefits. IAN model showed the best results for entity-level ADR classification task in all of our experiments.

There are three future research directions that require, from our point of view, more attention. First, we plan to add knowledge-based features as input for IAN model and evaluate their efficiency. Second, apply these models to the entitylevel ADR classification task for texts in other languages. Finally, we plan to explore the potential of new state-of-the-art text classification methods based on BERT language model.

\section{Acknowledgments}

This research was supported by the Russian Science Foundation grant no. 18-11-00284.

\section{References}

Ilseyar Alimova and Elena Tutubalina. 2017. Automated detection of adverse drug reactions from social media posts with machine learning. In International Conference on Analysis of Images, Social Networks and Texts, pages 3-15. Springer.

I.S. Alimova and E. V. Tutubalina. 2018. Entity-level classification of adverse drug reactions: a comparison of neural network models. Proceedings of the Institute for System Programming of the RAS, 30(5):177-196.

Nestor Alvaro, Yusuke Miyao, and Nigel Collier. 2017. Twimed: Twitter and pubmed comparable corpus of drugs, diseases, symptoms, and their relations. JMIR public health and surveillance, 3(2).

Eiji Aramaki, Yasuhide Miura, Masatsugu Tonoike, Tomoko Ohkuma, Hiroshi Masuichi, Kayo Waki, and Kazuhiko Ohe. 2010. Extraction of adverse drug effects from clinical records. In MedInfo, pages 739-743.

Adrian Benton, Lyle Ungar, Shawndra Hill, Sean Hennessy, Jun Mao, Annie Chung, Charles E Leonard, and John H Holmes. 2011. Identifying potential adverse effects using the web: A new approach to med- ical hypothesis generation. Journal of biomedical informatics, 44(6):989-996.

Jiang Bian, Umit Topaloglu, and Fan Yu. 2012. Towards large-scale twitter mining for drug-related adverse events. In Proceedings of the 2012 international workshop on Smart health and wellbeing, pages 25-32. ACM.

Shaika Chowdhury, Chenwei Zhang, and Philip S Yu. 2018. Multi-task pharmacovigilance mining from social media posts. arXiv preprint arXiv:1801.06294.

Hong-Jie Dai, Musa Touray, Jitendra Jonnagaddala, and Shabbir Syed-Abdul. 2016. Feature engineering for recognizing adverse drug reactions from twitter posts. Information, 7(2):27.

Shashank Gupta, Manish Gupta, Vasudeva Varma, Sachin Pawar, Nitin Ramrakhiyani, and Girish Keshav Palshikar. 2018a. Co-training for extraction of adverse drug reaction mentions from tweets. In $E u$ ropean Conference on Information Retrieval, pages 556-562. Springer.

Shashank Gupta, Sachin Pawar, Nitin Ramrakhiyani, Girish Keshav Palshikar, and Vasudeva Varma. 2018b. Semi-supervised recurrent neural network for adverse drug reaction mention extraction. $B M C$ bioinformatics, 19(8):212.

Rave Harpaz, Herbert S Chase, and Carol Friedman. 2010. Mining multi-item drug adverse effect associations in spontaneous reporting systems. In $B M C$ bioinformatics, volume 11, page S7. BioMed Central.

S. Hochreiter and J. Schmidhuber. 1997. Long ShortTerm Memory. Neural Computation, 9(8):17351780. Based on TR FKI-207-95, TUM (1995).

Yongfang Hou, Xinling Li, Guizhi Wu, and Xiaofei Ye. 2016. National adr monitoring system in china. Drug Safety, 39(11):1043-1051.

Binxuan Huang, Yanglan Ou, and Kathleen M Carley. 2018. Aspect level sentiment classification with attention-over-attention neural networks. In International Conference on Social Computing, Behavioral-Cultural Modeling and Prediction and Behavior Representation in Modeling and Simulation, pages 197-206. Springer. 
Trung Huynh, Yulan He, Alistair Willis, and Stefan Rüger. 2016. Adverse drug reaction classification with deep neural networks. In Proceedings of $\mathrm{COL}$ ING 2016, the 26th International Conference on Computational Linguistics: Technical Papers, pages 877-887.

V Ivanov, E Tutubalina, N Mingazov, and I Alimova. 2015. Extracting aspects, sentiment and categories of aspects in user reviews about restaurants and cars. In Proceedings of International Conference Dialog, volume 2, pages 22-34.

Jitendra Jonnagaddala, Toni Rose Jue, and Hong-Jie Dai. 2016. Binary classification of twitter posts for adverse drug reactions. In Proceedings of the Social Media Mining Shared Task Workshop at the Pacific Symposium on Biocomputing, Big Island, HI, USA, pages 4-8.

Sarvnaz Karimi, Alejandro Metke-Jimenez, Madonna Kemp, and Chen Wang. 2015. Cadec: A corpus of adverse drug event annotations. Journal of biomedical informatics, 55:73-81.

Svetlana Kiritchenko, Saif M Mohammad, Jason Morin, and Berry de Bruijn. 2018. Nrc-canada at smm4h shared task: Classifying tweets mentioning adverse drug reactions and medication intake. arXiv preprint arXiv:1805.04558.

Ioannis Korkontzelos, Azadeh Nikfarjam, Matthew Shardlow, Abeed Sarker, Sophia Ananiadou, and Graciela H Gonzalez. 2016. Analysis of the effect of sentiment analysis on extracting adverse drug reactions from tweets and forum posts. Journal of biomedical informatics, 62:148-158.

Siwei Lai, Liheng Xu, Kang Liu, and Jun Zhao. 2015. Recurrent convolutional neural networks for text classification. In $A A A I$, volume 333 , pages 2267 2273.

Xiao Liu and Hsinchun Chen. 2013. Azdrugminer: an information extraction system for mining patientreported adverse drug events in online patient forums. In International Conference on Smart Health, pages 134-150. Springer.

Dehong Ma, Sujian Li, Xiaodong Zhang, and Houfeng Wang. 2017. Interactive attention networks for aspect-level sentiment classification. arXiv preprint arXiv:1709.00893.

Z.Sh. Miftahutdinov, E.V. Tutubalina, and A.E. Tropsha. 2017. Identifying disease-related expressions in reviews using conditional random fields. Computational Linguistics and Intellectual Technologies: Papers from the Annual conference Dialogue, 1(16):155-166.

Diego Saldana Miranda. 2018. Automated detection of adverse drug reactions in the biomedical literature using convolutional neural networks and biomedical word embeddings. arXiv preprint arXiv:1804.09148.
Jin-Cheon Na, Wai Yan Min Kyaing, Christopher SG Khoo, Schubert Foo, Yun-Ke Chang, and Yin-Leng Theng. 2012. Sentiment classification of drug reviews using a rule-based linguistic approach. In International Conference on Asian Digital Libraries, pages 189-198. Springer.

Azadeh Nikfarjam and Graciela H Gonzalez. 2011. Pattern mining for extraction of mentions of adverse drug reactions from user comments. In AMIA Annual Symposium Proceedings, volume 2011, page 1019. American Medical Informatics Association.

Azadeh Nikfarjam, Abeed Sarker, Karen OConnor, Rachel Ginn, and Graciela Gonzalez. 2015. Pharmacovigilance from social media: mining adverse drug reaction mentions using sequence labeling with word embedding cluster features. Journal of the American Medical Informatics Association, 22(3):671-681.

Yun Niu, Xiaodan Zhu, Jianhua Li, and Graeme Hirst. 2005. Analysis of polarity information in medical text. In AMIA annual symposium proceedings, volume 2005, page 570. American Medical Informatics Association.

BAHADORREZA Ofoghi, SAMIN Siddiqui, and KARIN Verspoor. 2016. Read-biomed-ss: Adverse drug reaction classification of microblogs using emotional and conceptual enrichment. In Proceedings of the Social Media Mining Shared Task Workshop at the Pacific Symposium on Biocomputing.

Majid Rastegar-Mojarad, Ravikumar Komandur Elayavilli, Yue Yu, and Hongfang Liu. 2016. Detecting signals in noisy data-can ensemble classifiers help identify adverse drug reaction in tweets. In Proceedings of the Social Media Mining Shared Task Workshop at the Pacific Symposium on Biocomputing.

N. Rusnachenko and N. Loukachevitch. 2018. Using convolutional neural networks for sentiment attitude extraction from analytical texts. In Proceedings of CEUR Workshop, CLLS-2018 Conference.

Abeed Sarker, Rachel Ginn, Azadeh Nikfarjam, Karen OConnor, Karen Smith, Swetha Jayaraman, Tejaswi Upadhaya, and Graciela Gonzalez. 2015. Utilizing social media data for pharmacovigilance: a review. Journal of biomedical informatics, 54:202-212.

Abeed Sarker and Graciela Gonzalez. 2015. Portable automatic text classification for adverse drug reaction detection via multi-corpus training. Journal of biomedical informatics, 53:196-207.

Jesus Serrano-Guerrero, Jose A Olivas, Francisco P Romero, and Enrique Herrera-Viedma. 2015. Sentiment analysis: A review and comparative analysis of web services. Information Sciences, 311:18-38. 
SM Shareef, CDM Naidu, Shrinivas R Raikar, Y Venkata Rao, and U Devika. 2017. Development, implementation, and analysis of adverse drug reaction monitoring system in a rural tertiary care teaching hospital in narketpally, telangana. International Journal of Basic \& Clinical Pharmacology, 4(4):757-760.

Preeti Singh, Manju Agrawal, Rajesh Hishikar, Usha Joshi, Basant Maheshwari, and Ajay Halwai. 2017. Adverse drug reactions at adverse drug reaction monitoring center in raipur: Analysis of spontaneous reports during 1 year. Indian journal of pharmacology, 49(6):432.

V. Solovyev and V. Ivanov. 2014. Dictionary-based problem phrase extraction from user reviews. Lecture Notes in Computer Science (including subseries Lecture Notes in Artificial Intelligence and Lecture Notes in Bioinformatics), 8655 LNAI:225-232.

Youwei Song, Jiahai Wang, Tao Jiang, Zhiyue Liu, and Yanghui Rao. 2019. Attentional encoder network for targeted sentiment classification. arXiv preprint arXiv:1902.09314.

Yequan Wang, Minlie Huang, Li Zhao, et al. 2016. Attention-based lstm for aspect-level sentiment classification. In Proceedings of the 2016 conference on empirical methods in natural language processing, pages 606-615.

Lei Zhang, Shuai Wang, and Bing Liu. 2018. Deep learning for sentiment analysis: A survey. Wiley Interdisciplinary Reviews: Data Mining and Knowledge Discovery, page e1253.

Zhifei Zhang, JY Nie, and Xuyao Zhang. 2016. An ensemble method for binary classification of adverse drug reactions from social media. In Proceedings of the Social Media Mining Shared Task Workshop at the Pacific Symposium on Biocomputing.

Maryam Zolnoori, Kin Wah Fung, Timothy B Patrick, Paul Fontelo, Hadi Kharrazi, Anthony Faiola, Yi Shuan Shirley Wu, Christina E Eldredge, Jake Luo, Mike Conway, et al. 2019. A systematic approach for developing a corpus of patient reported adverse drug events: A case study for ssri and snri medications. Journal of biomedical informatics, 90:103091. 